\title{
Neonatal auditory brainstem response cannot reliably diagnose brainstem death
}

\author{
P R F DEAR AND D J GODFREY \\ Neonatal Unit, St James's University Hospital, Leeds
}

SUMmARY In two newborn infants who had experienced severe asphyxial insults, and who showed noticeable signs of brainstem dysfunction, all components of the auditory brainstem response except the eighth nerve potential became undetectable. Both babies survived, their brainstem responses returned, and one of them is judged to be developmentally normal at the age of 18 months. Clinical signs of brainstem dysfunction with complete cessation of conduction in the brainstem auditory pathway cannot be taken, therefore, as a sign of irreversible brainstem damage in the human newborn.

Advances in intensive care medicine during the past decade have dramatically reduced the neonatal mortality rate but may also have increased the risk of some babies surviving with severe central nervous system damage; and the dilemma of whether or not to continue life support when there is evidence suggesting the likelihood of serious neuropathology has probably become more common. Clinical evaluation of the immature nervous system requires a different approach from that appropriate to the older child or adult, and is in many ways less informative. Certainly the applicability of the current adult brain death criteria is in doubt.

There are good reasons to delay reaching a decision about terminating life support in these patients, since if the brainstem is dead cardiac arrest inevitably follows, and if there is severe damage to higher brain structures ventilatory support cannot usually be dispensed with for several days, during which time evidence about the nature and extent of the lesion may be accumulated by observation of clinical signs and ancillary investigations such as computed tomography, ultrasound, and electroencephalography.

The recent introduction into the neonatal field of neurophysiological tests based on the recording of electrical potentials from the brain evoked by sensory stimuli has raised the possibility of obtaining objective information about the functional integrity of certain brain structures. It is tempting to apply these tests to the difficult clinical situation under discussion in the hope that they may help in the making of clinical decisions. We wish to sound a note of caution regarding the interpretation of the auditory brainstem response in the neonatal period by reference to two patients that we have recently studied.

\section{Method}

Auditory brainstem response recording makes use of a computer averaging technique to isolate and amplify the tiny voltages emanating from structures in the auditory pathway in response to sound stimulation of the cochlea. The principles have been well described elsewhere ${ }^{1}$ and only aspects of our individual application of the technique will be described here.

The apparatus used to obtain the auditory brainstem response was a Medelec MS 92. Silver chloride disc electrodes $(0.5 \mathrm{~cm}$ diameter $)$ were used to record the far field brainstem potentials, and were placed on, or just ventral to, the anterior fontanelle (recording electrode) and over each mastoid process (reference and ground electrodes). The electrical resistance between paired electrodes was always reduced to less than $5 \mathrm{KOhm}$. Click stimuli of $0 \cdot 1$ milisecond duration were presented via a headphone to each ear in turn, at a repetition frequency of $30 \mathrm{~Hz}$. A sound intensity of $80 \mathrm{~dB}$ above normal adult hearing threshold was used in the patients reported here. Each recording, representing 10 miliseconds of time, was averaged from a minimum of 1500 clicks, and was only accepted if it could be exactly replicated at least once. Waveforms were identified and labelled according to the criteria of Jewett. ${ }^{1}$ No form of sedation was used. The technique is harmless and non-invasive and was approved by the hospital ethical committee.

During a two and a half year period the authors performed auditory brainstem response measurements on about 500 babies admitted to the neonatal unit. Among these were some 30 babies exhibiting frankly abnormal neurological signs after episodes 
of hypoxia, and in this group a variety of auditory brainstem response abnormalities have been discovered; ranging from slight prolongation of the latency of components of the waveform to complete absence of the auditory brainstem response. In only four patients have we been unable to detect any sign of the brainstem potentials and yet a clear wave I indicating activation of the cochlea and eighth nerve. It is with the two babies who survived after exhibiting this particular abnormality that this paper is concerned.

\section{Case histories and results}

Case 1. This girl was born at 37 weeks' gestation with a birthweight of $3700 \mathrm{~g}$. She was hydropic due to severe rhesus haemolytic disease (cord blood haemoglobin $3.5 \mathrm{~g} / 100 \mathrm{ml}$, cord blood bilirubin 190 $\mu \mathrm{mol} / \mathrm{l}(11 \cdot 1 \mathrm{mg} / 100 \mathrm{ml})$ and although there were no signs of life at birth, she responded to ventilation and cardiac massage. Her Apgar scores at 1 and 5 minutes were 0 and 4 respectively. She was ventilated from birth. An episode of cardiac arrest, from which she was successfully resuscitated, occurred during the first exchange transfusion at 2 hours of age. A second exchange transfusion was performed at 9 hours of age by which time she was drowsy and was having frequent tonic seizures. At 36 hours of age the auditory brainstem response at $80 \mathrm{~dB}$ was normal (Fig. 1) showing waves I (cochlea and eighth nerve), III (superior olivary complex), V (inferior colliculus), and VI (generator source uncertain). These waves were clear and reproducible and were at normal latencies. By 60 hours of age the baby had had a third exchange transfusion (at her maximum serum bilirubin of $280 \mu \mathrm{mol} / \mathrm{l}(16.4 \mathrm{mg} / 100 \mathrm{ml})$ ), was comatose, and apnoeic with an arterial $\mathrm{PCO}_{2}$ of $9 \mathrm{kPa}$. The corneal reflexes were absent and the anterior fontanelle was tense. An electroencephalogram at this time showed a burst suppression pattern. The auditory brainstem response at 60 hours of age (Fig. 1) is very abnormal, showing a wave I but no output from the brainstem.

She was extremely unwell for several days, with occasional seizures and a continuing need for ventilatory support. As she recovered consciousness she was at first very hypotonic with absent reflexes but later became hypertonic and irritable. Fig. 1 shows the gradual recovery of her auditory brainstem response to normal by 10 days of age. She has so far been followed up for a period of 18 months and her developmental progress is normal.

Case 2. This girl was born at 37 weeks' gestation with a birthweight of $3040 \mathrm{~g}$. She was noticeably hypotonic at birth, and for several days had

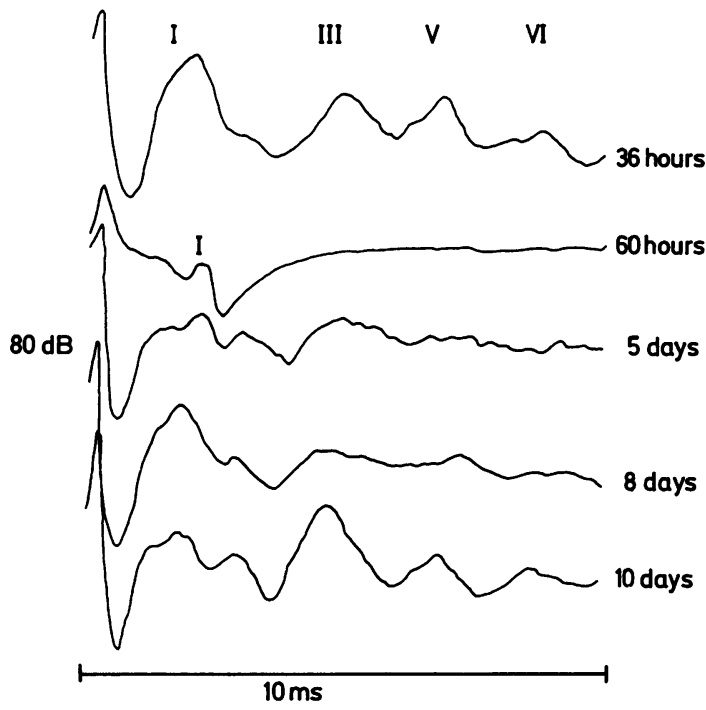

Fig. 1 Serial auditory brainstem response recordings from case 1. Each trace is electronically averaged from two separate recordings.

respiratory difficulty thought to be of central nervous system origin. She spent the first months of her life on the neonatal intensive care unit because of hypotonia, apathy, and poor feeding. Extensive investigations including computed tomography, electroencephalography, electromyography, muscle biopsy, and metabolic screening tests showed little. although the computed tomogram and electroencephalogram were compatible with hypoxic brain damage. Fig. 2 (top trace) shows her normal auditory brainstem response at 4 weeks of age. Three days after discharge home, at the age of 3 months, she was brought to casualty because of breathlessness and grunting respiration. On arrival she was pale and mottled, hypothermic and hypoxic, and had a cardiorespiratory arrest from which she was resuscitated. Chest radiograph showed a widespread pneumonia. Fig. 2 (middle pair of traces) shows her auditory brainstem response 24 hours after this episode, when she was on a ventilator and showing no spontaneous ventilatory effort at an arterial $\mathrm{PCO}_{2}$ of $6 \mathrm{kPa}$. Wave $\mathrm{I}$ is clearly visible, indicating eighth nerve activation, but no brainstem waves are visible. She recovered spontaneous breathing activity 36 hours later and came off mechanical ventilation after a further five days. Her subsequent clinical state, little different from that previously exhibited, was one of considerable apathy and hypotonia. Her auditory brainstem response one week after the described cardiorespiratory arrest is shown in Fig. 2 (bottom pair of 


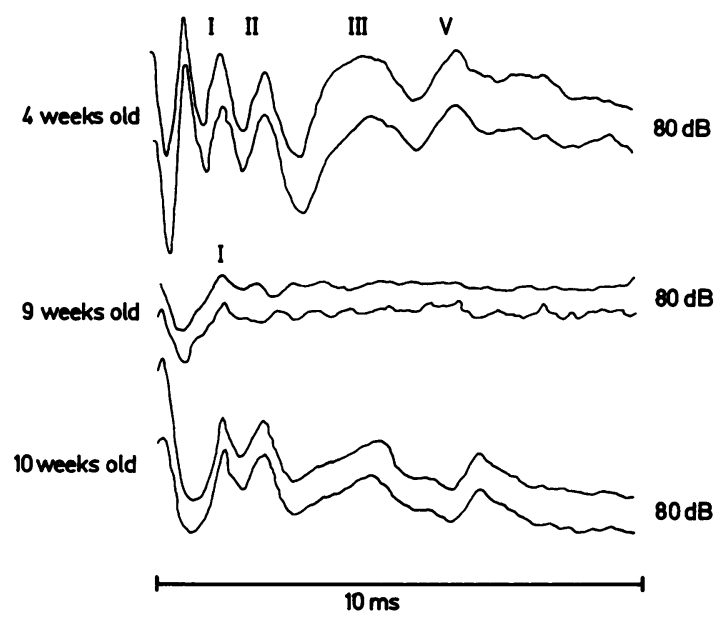

Fig. 2 Serial auditory brainstem response recordings from case 2. Each trace is electronically averaged from two separate recordings.

traces) and there has been substantial recovery, although the latencies of all the waves are prolonged compared with the first tracing at the age of 4 weeks. Unfortunately we have been unable to repeat her auditory brainstem response but she continues to show considerable developmental delay.

\section{Discussion}

The auditory brainstem response is a very suitable test for use in the newborn as it requires no cooperation from the patient other than that he or she be quiet for a few minutes. It provides information about the function of a neural pathway which extends through the brainstem, where so many vital centres are located, and the response is virtually uninfluenced by drugs or sleep state.

Our principle interest in the neonatal auditory brainstem response is as an indicator of maturation of brainstem conduction with increasing postconceptional age. Since the brainstem structures of the auditory pathway, however, are known to be susceptible to hypoxia in the newborn period, ${ }^{2}$ we extended the technique to babies who had had episodes of appreciable asphyxia or other central nervous system insults to see if it would be useful in detecting signs of brainstem injury.

It is important to note that in the patients we report a definite wave I was present, indicating activation of the cochlea and eighth nerve. We have seen other cases in which no auditory brainstem response components could be detected, but in the absence of a wave I the possibility of a lesion of the peripheral auditory apparatus cannot be excluded, and therefore no inference may be drawn about the function of the brainstem. This is especially important in the case of very immature infants who may not have yet developed detectable auditory brainstem responses. ${ }^{3}$

The pathophysiology underlying the transient disappearance of the auditory brainstem response waveforms, and in case 1 their gradual reappearance in a caudorostral progression, is a matter for speculation, but clearly this was something short of irreversible neuronal damage, possibly compression due to brainswelling. Bilirubin neurotoxicity in case 1 cannot be excluded, at least as a contributory factor, although in our experience to date a serum bilirubin of $280 \mu \mathrm{mol} / \mathrm{l}(16.4 \mathrm{mg} / 100 \mathrm{ml})$ in a baby of 37 weeks' gestation does not influence the auditory brainstem response.

Reports on the usefulness of the auditory brainstem response in assessing the prognosis for the unconscious adult patient ${ }^{4}{ }^{5}$ can be interpreted as showing a generally good correlation between absence of responses and a poor outcome, but by no means sufficiently good for the auditory brainstem response to predict accurately the outcome for any individual. We do not yet have sufficient data to make a general comment on the value of the auditory brainstem response as a prognostic indicator after insults to the central nervous system in the newborn period, but the discovery of two infants whose brainstems failed to conduct incoming eighth nerve impulses after asphyxial episodes, but who both survived-one seemingly neurologically intact-shows that at present the absence of the auditory brainstem response cannot be taken as evidence of irretrievable brainstem damage.

We thank Mr H A Beagley and Professor J A Davis for their help and encouragement.

\section{References}

Jewett DL, Williston JS. Auditory-evoked far fields averaged from the scalp of humans. Brain 1971;94:681-96.

2 Schneider H, Ballowitz L, Schachinger H, Hanefield F, Droszus JU. Anoxic encephalopathy with prominent involvement of the basal ganglia, brainstem and spinal cord in the perinatal period. Acta Neuropathol (Berl) 1975;32:287-98.

3 Roberts JL. Davis H. Phon GL, Reichert TJ, Sturtevant EM Marshall RE. Auditory brainstem responses in preterm neonates. Maturation and follow-up. J Pediatr 1982;101:257-63.

4 Starr A. Auditory brainstem responses in brain death. Brain 1976:99:543-54.

5 Goldic WD, Chiappa KH, Young RR, Brooks EB. Brainstem auditory and short-latency somatosensory evoked responses in brain death. Neurology (NY) 1981;31:248-56.

Correspondence to Dr P R F Dear, Neonatal Unit, St James's University Hospital, Leeds LS9 7TF.

Received 20 August 1984 